Indian J. Anim. Hlth. (2020), 59(2)-Special Issue: 27-35

DOI: $10.36062 /$ ijah.59.2SPL.2020.27-35

Invited Review Article

\title{
ROLE OF GUT MICROBIOTA IN VIRAL INFECTIONS
}

\author{
S. SARKAR ${ }^{1 *}$ AND M. BHOWMIK ${ }^{2}$ \\ ${ }^{1}$ Department of Genetics, School of Medicine, The University of North Carolina at Chapel Hill, NC \\ 27599, USA \\ ${ }^{2}$ Department of Zoology, The University of Burdwan, West Bengal- 713 104, India
}

\begin{abstract}
Microbiome is a complex network of thousands of microorganisms including bacteria, viruses, fungi and other microorganisms that remain as commensals. These microorganisms maintain an intricate balance with hosts' immune system to stop overexploitation of hosts' resources while shaping the host immune system by priming the adaptive immune system for optimal development. Viruses are the obligatory intracellular parasites that depend on the host-cell machinery for their survival, genome replication, and producing progeny virions. The first key step in the virus life cycle is the entry during which the virus comes in contact with hosts' skin or mucous membrane where hundreds of thousands of microorganisms colonize. As such, there is a possibility of complex interaction of invading viruses and other microorganisms within the microbiome, for example symbionts bacteria may interact with viruses and also immune system maintaining the homeostasis within hosts. Although the interaction between viruses and the microbiome are not very clear it is possible that the microbiota, especially the bacteria prime the immune system and confer a resistance to the invading viruses to initiate an infection or a disease. It may also be possible that the bacterial components of the microbiome actually initiate an immunosuppressive reaction that may enhance viral infection or pathogenesis. In this review, we have highlighted the various outcomes that may happen due to the complex interaction of the microbiota and viruses and have also discussed how the viral entry, replication and/or pathogenesis are modulated by microbiota.
\end{abstract}

Key words: Host-pathogen, Intestinal microbiota, Microbiome, Virome, Virus

\section{Introduction}

The human as well as the animal body surfaces such as skin and body cavities are colonized by a humongous number of symbiotic commensal microorganisms, collectively known as microbiota. The human body comprises of about 100 trillion cells of which these microorganisms outnumbers their host cells at least by a factor of 10 and also express at least 10-fold more unique genes compared to their hosts (Ley et al., 2006). These large number of microorganisms which include bacteria, viruses, fungi, and other eukaryotic species maintain a homeostatic relationship through the complex interaction with their host cells. These enormous microbiomes provide the host with tremendous enzymatic capabilities regulating many aspects of hosts physiology (Belkaid and Hand, 2014). In fact, with the advent of modern technology, advanced bioinformatics and culture-independent genomic techniques, microbiome research has been revolutionized over the past few years and it is now evident that these microorganisms just do not colonize hosts as passive bystanders rather actively playing fundamental roles in shaping host health and immunity, maintaining

\footnotetext{
*Corresponding Author
} 
multiple aspects of host physiology, but not limited to digestion, metabolism, nutritional responses, circadian rhythm, and disease pathogenesis (Hacquard et al., 2015; Lynch and Hsiao, 2019).

Viruses are the obligatory parasites that require host cellular machinery for their own replication and survival. Although most of the viruses are identified on the basis of their mechanism of pathogenesis, many more viruses are found within a healthy individual that actually do not cause disease (Domínguez-Díaz et al., 2019). These viruses that reside within the body cavities as part of microbiome are collectively known as virome. These consist of all viruses of the prokaryotic and the eukaryotic organisms found in humans and animals. Although, at this moment the role of the virome in health and disease is very poorly understood, it is clear that the non-pathogenic viruses within microbiome remain mainly as commensals with their hosts along with bacteria and other microorganisms. They have co-evolved with their host for years maintaining a homeostasis, (Dethlefsen et al., 2007). These intricate, perfectly balanced homeostatic relationships, require an optimally functioning immune system which is a complex network of innate and adaptive components that prevents the microorganisms from over-exploitation of their host resources (Zheng et al., 2020). On the other hand, perturbations in the balance of the microbiome communities by different stimuli such as environmental factor including use of antibiotics, changes in diet or geography, and alterations of the immune function may lead to the systemic dissemination of the apparently harmless commensal microorganisms within hosts, enhanced susceptibility to pathogenic organisms resulting in alteration in disease severity and overall health status of the hosts (Zheng et al., 2020). The alteration of microbiome however, may also provide resistance to some microorganisms including viruses. The first key step in the virus life cycle is the entry into the host, during which the virus comes in contact with hosts' skin or mucous membrane where numbers of microorganisms colonize. As such, there is a possibility of complex interaction of invading viruses and other microorganisms, for example, commensal bacteria may interact with viruses and also immune system resulting in the alteration of the intricate homeostatic balance. Although the impact of this interaction between viruses and the microbiome are not very clear, however, it is possible that the microbiota, especially the bacteria primes the immune system and confers resistance to the invading viruses to initiate an infection or a disease. It may also be possible that the bacterial components of the microbiome actually initiate an immunosuppressive reaction that may enhance viral infection or pathogenesis. In fact, multiple recent studies have reported that disease pathogenesis caused by various human and animal viruses can be modulated by the normal intestinal microbiota of the hosts (Wilks and Golovkina, 2012; Karst, 2016). In this review we have discussed about the role of microbiota in the development of immune system, general health status of humans and animals and role of microbiota in the viral infections and their pathogenesis. We have also discussed the various outcomes that may happen due to the complex interaction of the microbiome, host and viruses and have pointed out how the viral infections and/or pathogenesis are modulated by microbiota.

\section{Small animal model to study gut microbiome} For the infectious disease research and the preclinical evaluation of vaccines and therapies against many human pathogens, small animal models such as mice are one of the most important tools (Sarkar and Heise, 2019). Similarly, mouse models serve as invaluable tool for studying microbiome, especially intestinal microbiome. Scientists have adopted two important strategies to study the effect of 
gut microbiota on disease pathogenesis including metabolic diseases, cancer, bacterial diseases or viral diseases. One strategy is the infection of germ-free or sterile mouse with the pathogens in question and the other strategy is the infection of mouse that are treated with a cocktail of oral antibiotics/antimicrobials to deplete of their natural microbiota in the intestine. Both of the models have pros and cons. For biomedical research purposes majority of the mouse that are used, are specific pathogen free (SPF) but not completely devoid of all microorganisms. On the other hand, germ-free mice are bred and raised in a sterile condition; hence, are free from all microorganisms, including the commensal intestinal microbiota (Karst, 2016). As such, these germ-free mice can be easily used to investigate the effect of the commensal gut microbiota on the pathogenesis of a particular virus, bacteria or any other pathogens. These mice can also be used to study the effect of gut microbiota on the homeostatic condition of the host. However, because of complete absence of all commensal microbiota in the intestine, that otherwise play an important role in the development of mucosal immunity, the germfree mice have many abnormalities both in the morphology of the intestine as well as in the mucosal immunity (Karst, 2016).

In the antibiotic-depletion method, mice are treated with oral gavage containing either broad-spectrum antibiotics or a cocktail of antibiotics for example ampicillin, vancomycin, neomycin, metronidazole and amphotericin $\mathrm{B}$ daily for a few consecutive days (time depends on the study) targeting both Gram-positive and Gram-negative bacteria (Karst, 2016; Sun et al., 2019). After a certain time of post-gavage treatment, antibiotics are added in the drinking water and then the efficiency of the microbiotadepletion is assessed by culturing the feces for microorganisms in anaerobic followed by aerobic conditions (Karst, 2016). The use of antibiotic depletion method has advantage over the use of germ-free mice, as in the antibioticdepletion methods, mice do not require a special handling in sterile condition such as using sterile isolators. Further, it also caused to increase the cost of mouse-breeding, maintenance and overall research as well as mice with antibiotic treatment will also have normal mucosal immune system. Although majority of the commensal microbiota from intestine are removed, antibiotic-depletion cannot remove all intestinal commensal microbiota; hence, researchers must account for the effect of the residual microbiota on the questions they ask (Karst, 2016).

\section{Modulation of the viral infections by microbiota}

It has now been shown that the fate of the many pathogenic viral infections depends on the nature of the microbiota present in the hosts and especially microbiota present in the intestine which is the richest source of microbial community among others (Karst, 2016). Generally, microbiota can influence viral infections in three different ways. Firstly, the commensal microbiota provides protective immunity against invading pathogenic viruses either by priming immune system or working as a physical barrier to viruses for entry to host cells (Kasubuchi et al., 2015; Winkler et al., 2020). Secondly, the microbiota can directly enhance the invading viral infections (Kane et al., 2011; Karst, 2016). Thirdly, the commensal microbiota can indirectly promote viral infections (Isaak et al., 1988).

Gut microbiome promoting resistance to viral infections: There is no doubt that intestinal microbiota interacts with invading pathogens whether it is virus or bacteria, modulates the host's immune system, and thus modulates the disease pathogenesis. In fact, multiple evidence suggests that gut microbiota maintains a mutualistic relationship that benefits their 
human host in many ways (Karst, 2016). These commensal microbiota for example would degrade the otherwise indigestible dietary fibers resulting in the production of short-chain fatty acids (SCFAs) such as acetate, propionate, and butyrate (Flint et al., 2008; Kasubuchi et al., 2015). These SCFAs are then used for the de novo synthesis of the end products like lipids and glucose which serve as the energy source for hosts' cellular metabolism and regulators of gut motility (Tremaroli and Bäckhed, 2012). The gut microbiota is also involved in the development of the mucosal immune system, as evident from the inability of the germ-free mice to mount an optimal immune response including the reduction in the secretion of immunoglobulin $\mathrm{A}$ and intestinal $\mathrm{T}$ cells because of the impaired development of mature lymphoid organs within the gastrointestinal tract (Kamada and Núñez, 2014). Certain intestinal microorganisms especially bacteria can induce the secretion of antimicrobial proteins like angiogenin and the C-type lectin RegIII $\gamma$ by intestinal epithelial cells which in turn protect the hosts from invading pathogenic bacteria (Hooper et al., 2012). However, gut microbiota can also provide hosts protection against invading pathogenic viruses including influenza, dengue, human immunodeficiency virus (HIV), and chikungunya virus among others. Intestinal microbiota not only regulates immune homeostasis in intestine but also regulate immune response in other mucosal surfaces such as in the respiratory tract. It has been observed that the gut microbiome critically regulates the development of influenza-specific $\mathrm{CD}^{+}$and $\mathrm{CD}^{+} \mathrm{T}$ cell responses as well as the virus-specific antibody-mediated immune responses (Ichinohe et al., 2011). In their mouse model, they have shown that there is impaired generation of influenza-specific $\mathrm{CD}^{+}$, $\mathrm{CD}^{+} \mathrm{T}$ cell responses and also antibody $(\operatorname{IgA})$ mediated immune responses when natural commensal microbiota especially neomycinsensitive bacteria were depleted by the use of oral antibiotic treatment (Ichinohe et al., 2011). The neomycin-sensitive commensal bacteria in the intestine provided signals for robust priming of pro-IL-1 $\beta$ and pro-IL-18 expression at steady state which provided immunocompetence in the lung (Ichinohe et al., 2011). Either local or distal injection of Toll-like receptor ligands rescued the impaired virusspecific immune responses (Ichinohe et al., 2011). In another study, Abt and associates have documented an exacerbation of influenza disease pathogenesis both in antibiotic-treated commensal microbiota-depleted SPF as well as germ-free or gnotobiotic mice (Abt et al., 2012). In the microbiota-depleted influenzainfected mice, the viral titers were significantly higher with an increased mortality rate compared to control SPF mice with normal intestinal microbiota. The histological analyses found morphological features of bronchiole epithelial degeneration and necrosis in the microbiota-depleted mice correlating the disease severity (Abt et al., 2012). Similar findings such as increased viral titers in the lung, exacerbated pathology and decreased survival rate were found in the germ-free mice when infected with influenza virus suggesting that intestinal commensal microbiota provides resistance against influenza virus infection in experimental animals (Abt et al., 2012). However, the increased disease severity in the sterile or microbiota-depleted mice may also be occurred due to the impaired protective mucosal immunity in these mice.

Chikungunya virus (CHIKV), which is an arthropod-borne (Arbo) emerging alphavirus, has infected millions of people around the globe posing a threat worldwide along with other emerging alphaviruses (Gunn et al., 2018; Broeckel et al., 2019; Nguyen et al., 2019). In a recent study, scientists have shown that gut microbiota plays a crucial role in the disease pathogenesis and protection against CHIKV. When infected with CHIKV, both germ-free 
mice and antibiotics treated, microbiotadepleted SPF mice either completely or partially devoid of intestinal microbiota, have shown an exacerbated disease pathogenesis producing early clinical signs, higher viral burdens in serum, spleen and contralateral ankle tissues, and also enhanced dissemination compared to SPF mice with normal intestinal microbiome (Winkler et al., 2020). The absence of microbiota or alteration of gut microbiota in mice impairs the TLR7-MyD88 signaling in plasmacytoid dendritic cells (pDCs) and suppresses systemic production of type I interferons (IFNs) resulting in the expression of fewer IFN-stimulated genes (ISGs) in the circulating monocytes making them more permissive for CHIKV infection (Winkler et al., 2020). Furthermore, they have demonstrated that the reconstitution of mice gut with a single species of bacteria, Clostridium scindens, or its derived metabolite, the secondary bile acid deoxycholic acid, can actually restore pDC- and MyD88-dependent type I IFN responses to protect mice from systemic CHIKV infection and transmission back to mosquito vectors (Winkler et al., 2020).

Another important arbovirus is dengue which belongs to the family Flaviviridae, infects millions of people around the world every year with significant morbidity and mortality. Dengue virus (DENV) is transmitted predominantly by the mosquito, Aedes aegypti which takes up the virus in their blood meal from an infected host (Ramirez et al., 2012). The virus infects the mid-gut of the mosquitoes, replicates there and migrates towards the salivary glands from where it can again be transmitted to a new host during another blood meal from an uninfected host. During this process of infecting a human host the virus actually comes in the vicinity of the microbiota present in the environment of mosquito midgut which has the possibility of modulating the Dengue fever disease pathogenesis. In fact, it has been shown experimentally that depletion of commensal gut microbiota of Aedes aegypti mosquitoes with antibiotic treatment, caused enhanced DENV replication with significantly higher titers and when mosquito-microbiota were reconstituted with a single isolate of Proteus sp. it mitigated the virus replication in the mosquitoes (Ramirez et al., 2012). Their studies indicated that gut microbiota of the mosquitoes promoted a basal level stimulation of the MyD88-dependent Toll pathway in response to DENV infection providing an antiviral immunity to DENV (Xi et al., 2008; Ramirez et al., 2012).

Like gut microbiome, vaginal microbiome also plays an important role in providing immunity or resistance to viral infection as has been seen in the case of vaginal human immunodeficiency virus-1 (HIV-1) infection in women. Alteration of vaginal microbiota increases the risk of acquiring and transmitting HIV-1 infection. In women, the vaginal microbiota is dominated by the presence of Lactobacilli that generate a byproduct, L-lactic acid which acidifies the vagina serving as antimicrobial agent (Lai et al., 2009; Ravel et al., 2011). L-lactic acid has been shown to function as broad-spectrum HIV virucide (both anti-HIV-1 and anti-HIV-2) in vitro conditions (Lai et al., 2009; Ravel et al., 2011). L-lactic acid mediated acidified mucous of the cervix and vagina can slow down the diffusion of HIV particle by trapping them within the acidified mucous at acidic $\mathrm{pH}$ which in turn reduces the acquisition and transmission risk of HIV infection (Lai et al., 2009).

\section{Gut microbiome promoting viral infections:}

Although commensal microbiota can provide resistance or antiviral immunity to many viral infections, it can also promote invading pathogenic viral infections both directly and indirectly. The first important study which demonstrated that gut microbiota enhances pathogenic viral infections included poliovirus, 
reovirus and mouse mammary tumor virus (MMTV) (Kane et al., 2011; Kuss et al., 2011; Karst, 2016). Antibiotic-treated mice depleted of commensal gut microbiota showed an attenuation of poliovirus infection compared to mice with normal microbiome (Kuss et al., 2011). Interestingly, reconstitution of the gut microbiota in the antibiotic-treated mice restored the pathogenesis of poliovirus indicating that intestinal microbiome enhances poliovirus infection (Kuss et al., 2011). Similar findings were documented in the reovirus mouse model of IFN-deficient mice which are normally susceptible to the infection but when mice were treated with antibiotics to deplete its natural microbiota and infected with reovirus, the mice failed to produce the disease. Tissue pathologies and fecal abnormalities suggested a role of intestinal microbiome in reovirus infection and pathogenesis (Kuss et al., 2011). Another group of scientists also conducted a study where they had taken either germ-free mice or SPF mice treated with antibiotics and infected with MMTV. These MMTV-infected mice failed to transmit the virus to their offspring through milk and a simple restoration of intestinal microbiota restored the transmission capabilities of the mother to their pups, corroborating the role of intestinal microorganisms enhancing the viral infections (Kane et al., 2011). Very similar observations were made in rotavirus and murine norovirus infections where microbiome plays crucial role in the promotion of viral infection and pathogenesis of the diseases. When antibiotic treated microbiota-depleted mice were infected with rotavirus, the mice produced an attenuated disease signs with reduced level of viral antigen in feces, reduced levels of viral genomes in intestinal tissue and delayed viral shedding when compared with control mice (Uchiyama et al., 2014). Moreover, antibiotic-treated rotavirus-infected suckling mice showed reduced incidence as well as duration of the diarrhea further establishing the role of gut microbiome in enhancing the viral infections (Uchiyama et al., 2014). Multiple studies have also found that intestinal microbiome promotes murine norovirus infections. Using either gnotobiotic mice or SPF-mice treated with oral antibiotics, it has been shown that the absence of gut microbiota attenuates norovirus pathogenesis by reducing in vivo virus replication, and virus shedding through feces (Jones et al., 2014; Kernbauer et al., 2014; Baldridge et al., 2015). These studies further demonstrate that intestinal microbiota is not only maintaining homeostasis in the host, rather can actually enhance a diverseset of viral infections and disease pathogenesis.

\section{Indirect mechanism of enhancing microbiome-mediated viral infections: The} intestinal microbiota can enhance invading viral infections in multiple ways. One example is the enhancement of viral infections by stimulating the activation and/or proliferation of the target cells which has been reported for the retroviruses that infect proliferating cells such as murine leukemia virus (MuLV) (Isaak et al., 1988). Germ-free mice infected with MuLV have failed to produce leukemia which was thought to be due to the absence of microbiota mediated lymphoid cell proliferation (Isaak et al., 1988). The gut microbiota can also enhance viral infections indirectly, specifically of enteric viruses by modulating antiviral immune responses, either by inducing a tolerogenic microenvironment rendering viral evasion of the host immune system, or by suppressing the production of virus-specific antibodies or also by altering the IFN-mediated signaling (Karst, 2016).

\section{Direct mechanism of enhancing microbiome-mediated viral infections: The} intestinal microbiome can directly enhance the infection of many invading viral pathogens in several ways including by virion stabilization further promoting viral transmissibility and by 
promoting the virus attachment to host cells (Karst, 2016). It has been shown that intestinal commensal bacteria helps in the stabilization of poliovirus particle and enhances its infection of host cells (Robinson et al., 2014). Interestingly, this stabilization by bacteria did not require the live bacteria, rather just the bacterial membrane component, lipopolysaccharides (LPS) and other Nacetylglucosamine-containing bacterial surface polysaccharides that were longer than six monosaccharides, were sufficient to provide this effect of virion stabilization (Robinson et al., 2014). This phenomenon is thought to be mediated by a direct interaction of poliovirus virion and LPS or other N- acetylglucosamine (Robinson et al., 2014). The poliovirus interaction with either bacteria or bacterial surface polysaccharides increases the thermostability and resistance of virus to inactivation by dilute chlorine bleach (Robinson et al., 2014). In addition to the virion stability, gut microbiota also promotes virus attachment as has been shown for poliovirus (Robinson et $a l ., 2014)$. The bacterial LPS also enhances poliovirus attachment to the surface of target cells facilitating the viral binding to the poliovirus receptor resulting in the enhanced infection (Robinson et al., 2014). The similar findings were documented in the human norovirus infection where an enhanced infection of B cells with norovirus was found in the presence of Enterobacter cloacae that express the H-type histo-blood group antigen (HBGA) but not H-type-negative, Escherichia coli strain (Tan and Jiang, 2005; Karst, 2015).

In summary, host microbiome can interact with the components of the immune system, actively shapes the immune system, and modulates the immune system either in the favor or against of the host depending on the interacting microbes. This in turn either protects the host from the invading pathogenic virus infections or enhances the severity of the viral infections directly or indirectly.

\section{Conclusion}

There have been significant discoveries in the field of microbiome within the last few years and a plethora of information are available describing the importance of microbiome in human and animal health. It is now clear that the tremendous number of microorganisms that reside within the bodies as commensals are not just passively present there, rather they are actively involved in the development of immune system, priming the immune system, modulating them to regulate host response to various pathogens. However, to date very little is known about the broad picture of the contribution of the microbiome in human and animal health. So far whatever the information is known primarily highlights the role of bacteria as commensals and their role in human and animal health. We now know that commensal bacteria both in intestine and other areas of the body cavities actively take part in modulating the pathogenesis of many pathogenic bacteria as well as viruses. It is very interesting to note that microbiota especially bacteria directly or indirectly interacts with viruses or host immune system and influences the outcome as either protective or detrimental for the host. While the commensal microbiota positively manipulates the host adaptive immune system in favor of hosts and thus provides a protective immunity against viruses like influenza, dengue, chikungunya viruses or HIV, microbiota also interacts with different components of immune system or directly with invading viruses like poliovirus, MuLV, norovirus, reovirus and others favoring the pathogens resulting an increased virus replication, dissemination and overall disease severity. It is thus imperative that researchers are focused to understand this complex microbiota-virus-host interaction in more details to identify a therapeutic approach. For example, by identifying the bacterial components required in virus infection as is shown for poliovirus requirement for bacterial surface polysaccharides, a therapeutic intervention may be targeted. As the dysregulation or ablation of commensal 
organisms enhances disease severity as in the case of influenza infection, it is worth evaluating the consequences and benefits of administering antibiotic therapy in severe cases of influenza with bacterial co-infections and in general use of antibiotics should be done with more cautions. However, further research is required to reveal more about the complex hostmicrobiota-virus interactions which will undoubtedly provide more opportunities to design novel therapies against many pathogenic viruses and bacteria. It is also interesting to note that besides bacteria, host

\section{REFERENCES}

Abt MC, Osborne LC, Monticelli LA, Doering TA, Alenghat $\mathrm{T}$ et al., 2012. Commensal bacteria calibrate the activation threshold of innate antiviral immunity. Immunity, 37: 158-170, doi: 10.1016/j.immuni.2012.04.011

Baldridge MT, Nice TJ, McCune BT, Yokoyama CC, Kambal A et al., 2015. Commensal microbes and interferon- $\lambda$ determine persistence of enteric murine norovirus infection. Science, 347: 266269, doi: $10.1126 /$ science. 1258025

Belkaid Y and Hand TW, 2014. Role of the microbiota in immunity and inflammation. Cell, 157: 121141, doi: 10.1016/j.cell.2014. 03.011

Broeckel R, Sarkar S, May NA, Totonchy J, Kreklywich CN et al., 2019. Src family kinase inhibitors block translation of alphavirus subgenomic mrnas. Antimicrob Agents Chemother, 63(4): e0232518, doi: 10.1128/AAC.02325-18

Dethlefsen L, McFall-Ngai M and Relman DA, 2007. An ecological and evolutionary perspective on human-microbe mutualism and disease. Nature, 449: 811-818, doi: 10.1038/nature06245

Domínguez-Díaz C, García-Orozco A, Riera- Leal A, Padilla-Arellano JR and Fafutis-Morris M, 2019. Microbiota and its role on viral evasion: is it with us or against us? Front Cell Infect Microbiol, 9: 256, doi: 10.3389/fcimb .2019.00256

Flint HJ, Bayer EA, Rincon MT, Lamed R and White BA, 2008. Polysaccharide utilization by gut bacteria: potential for new insights from genomic analysis. Nat Rev Microbiol, 6: 121-131, doi: 10.1038/nrmicro1817 microbiome also contains a very large number of commensal viruses and very little or no information is available about the importance of the virome if any in the pathogenesis of invading microorganisms. Probably, with the advent of modern technologies, next generation sequencing facilities and bioinformatics future studies will be conducted and more information will be available identifying the role of virome in the microbiome in the overall health of hosts.

Conflict of interest: The authors declare no conflict of interest.

Gunn BM, Jones JE, Shabman RS, Whitmore AC, Sarkar S et al., 2018. Ross River virus envelope glycans contribute to disease through activation of the host complement system. Virology, 515: 250-260, doi: $10.1016 /$ j.virol.2017.12.022

Hacquard S, Garrido-Oter R, González A, Spaepen S, Ackermann $\mathrm{G}$ et al., 2015. Microbiota and host nutrition across plant and animal kingdoms. Cell Host Microbe, 17: 603-616, doi: 1016/j.chom. 2015.04.009

Hooper LV, Littman DR and Macpherson AJ, 2012. Interactions between the microbiota and the immune system. Science, 336: 1268-1273, doi: 10.1126/science. 1223490

Ichinohe T, Pang IK, Kumamoto Y, Peaper DR, Ho JH et al., 2011. Microbiota regulates immune defense against respiratory tract influenza $A$ virus infection. Proc Natl Acad Sci, USA, 108: 53545359, doi: 10.1073/pnas. 1019378108

Isaak DD, Bartizal KF and Caulfield MJ, 1988. Decreased pathogenicity of murine leukemia virus-Moloney in gnotobiotic mice. Leukemia, 2:540-544

Jones MK, Watanabe M, Zhu S, Graves CL, Keyes LR et al., 2014. Enteric bacteria promote human and mouse norovirus infection of B cells. Science, 346: 755-759, doi: $10.1126 /$ science. 1257147

Kamada N and Núñez G, 2014. Regulation of the immune system by the resident intestinal bacteria. Gastroenterology, 146: 1477-1488, doi: 10.1053/ j.gastro.2014.01.060

Kane M, Case LK, Kopaskie K, Kozlova A, MacDearmid C et al., 2011. Successful transmission of a retrovirus depends on the 
commensal microbiota. Science, 334: 245-249, doi: $10.1126 /$ science. 1210718

Karst SM, 2015. Identification of a novel cellular target and a co-factor for norovirus infection - B cells and commensal bacteria. Gut Microbes, 6: 266-271, doi: 10.1080/19490976.2015. 1052211

Karst SM, 2016. The influence of commensal bacteria on infection with enteric viruses. Nat Rev Microbiol, 14: 197-204, doi: 10.1038/nrmicro.2015.25

Kasubuchi M, Hasegawa S, Hiramatsu T, Ichimura A and Kimura I, 2015. Dietary gut microbial metabolites, short-chain fatty acids, and host metabolic regulation. Nutrients, 7: 2839-2849, doi: 10.3390/ nu7042839

Kernbauer E, Ding Y and Cadwell K, 2014. An enteric virus can replace the beneficial function of commensal bacteria. Nature, 516: 94-98, doi: 10.1038/nature13960

Kuss SK, Best GT, Etheredge CA, Pruijssers AJ, Frierson JM et al., 2011. Intestinal microbiota promote enteric virus replication and systemic pathogenesis. Science, 334: 249-252, doi: 10.1126/science. 1211057

Lai SK, Hida K, Shukair S, Wang YY, Figueiredo A et al., 2009. Human immunodeficiency virus type 1 is trapped by acidic but not by neutralized human cervicovaginal mucus. J Virol, 83: 11196-11200, doi: 10.1128/JVI.01899-08

Ley RE, Peterson DA and Gordon JI, 2006. Ecological and evolutionary forces shaping microbial diversity in the human intestine. Cell, 124: 837-848, doi: 10.1016/j.cell.2006. 02.017

Lynch JB and Hsiao EY, 2019. Microbiomes as sources of emergent host phenotypes. Science, 365: 14051409, doi: 10.1126/science.aay0240

Nguyen TH, Haese NN, Madadi N, Sarkar S, Bonin Ket al., 2019. Studies on dibenzylamines as inhibitors of Venezuelan equine encephalitis virus. ACS Infect Dis, 5: 2014-2028, doi: 10.1021/acsinfecdis. 9b00035

Ramirez JL, Souza-Neto J, Torres Cosme R, Rovira J, Ortiz A et al., 2012. Reciprocal tripartite interactions between the Aedes aegypti midgut microbiota, innate immune system and dengue virus influences vector competence. PloS Negl Trop Dis, 6: e1561, doi: 10.1371/journal.pntd.0001561
Ravel J, Gajer P, Abdo Z, Schneider GM, Koenig SSK et al., 2011. Vaginal microbiome of reproductive-age women. Proc Natl Acad Sci, USA, 108(Suppl1): 4680-4687, doi: 10.1073/pnas.1002611107

Robinson CM, Jesudhasan PR and Pfeiffer JK, 2014. Bacterial lipopolysaccharide binding enhances virion stability and promotes environmental fitness of an enteric virus. Cell Host Microbe, 15: 36-46, doi: 10.1016/j.chom.2013.12.004

Sarkar S and Heise MT, 2019. Mouse models as resources for studying infectious diseases. Clin Ther, 41: 1912-1922, doi: 10.1016/j.clinthera. 2019.08.010

Sun L, Zhang X, Zhang Y, Zheng K, Xiang Q et al., 2019. Antibiotic-induced disruption of gut microbiota alters local metabolomes and immune responses. Front Cell Infect Microbiol, 9: 99, doi:10.3389/ fcimb.2019.00099

Tan M and Jiang X, 2005. Norovirus and its histo-blood group antigen receptors: an answer to a historical puzzle. Trends Microbiol, 13: 285-293, doi: 10.1016/j.tim.2005.04.004

Tremaroli V and Bäckhed F, 2012. Functional interactions between the gut microbiota and host metabolism. Nature, 489: 242-249, doi: 10.1038/ nature 11552

Uchiyama R, Chassaing B, Zhang B and Gewirtz AT, 2014. Antibiotic treatment suppresses rotavirus infection and enhances specific humoral immunity. J Infect Dis, 210: 171-182, doi: 10.1093/infdis/jiu037

Wilks J and Golovkina T, 2012. Influence of microbiota on viral infections. PLoS Pathog, 8: e1002681, doi: 10.1371/journal. ppat. 1002681

Winkler ES, Shrihari S, Hykes BL, Handley SA, Andhey PS et al., 2020. The intestinal microbiome restricts alphavirus infection and dissemination through a bile acid-type I IFN signaling axis. Cell, 182: 901918, doi: 10.1016/j.cell.2020.06.029

Xi Z, Ramirez JL and Dimopoulos G, 2008. The Aedes aegypti toll pathway controls dengue virus infection. PLoS Pathog, 4: e1000098, doi: 10.1371/journal.ppat. 1000098

Zheng D, Liwinski T and Elinav E, 2020. Interaction between microbiota and immunity in health and disease. Cell Res, 30: 492-506, doi: 10.1038/ s41422-020-0332-7

Received-12.10.2020, Accepted - 24.11.2020, Published - 01.12.2020 